\title{
Towards a New Dimension for User Modeling: The Use of Sensory Vocabulary'
}

\author{
Gudrun Kellner ${ }^{1}$, Bettina Berendt ${ }^{2}$ \\ ${ }^{1}$ Vienna University of Technology, Institute of Software Technology and Interactive Systems, \\ Favoritenstr. 9-11/188, 1040 Vienna, Austria. gudrun.kellner@tuwien.ac.at \\ ${ }^{2}$ K.U. Leuven, Department of Computer Science, \\ Celestijnenlaan 200A, 3001 Leuven-Heverlee, Belgium. bettina.berendt@cs.kuleuven.be
}

\begin{abstract}
One aspect of user preference, which is of high interest especially for the field of e-learning, concerns the mode of presenting information: What sensory system(s) should be addressed to make information interesting and easy to understand for the user? The answer might be found when looking at the user's perceptual preferences. But how to elicit those preferences? To test the user would be the most direct way - and the most annoying for the user.

In our research, we investigate the use of sensory vocabulary in forum texts as a source for implicit information on the user. Therefore, a corpus with more than $1,000,000$ forum posts was analyzed for the occurrence of expressions that are directly linked to a sensory system. We found that users differ significantly in their use of sensory expressions and that most users have preferred patterns for the use of sensory expressions.
\end{abstract}

Keywords: User Modeling, Perception, Language use

\section{Introduction}

Digital media permits to present information in manifold ways. An adequate mode of information presentation helps the user with information processing, e.g. user A prefers to read new information whereas user B prefers listening to it. Research shows that information can be more easily understood if its presentation is adapted to the cognitive style of the target user [5]. Cognitive style "may be defined as an individual's consistent approach to organising and processing information during thinking" [14]. In education, cognitive or learning styles describe different preferences in how learners perceive and retain information. One aspect of cognitive preference consists in the preferred mode of perception $[4 ; 5 ; 6 ; 14]$. Such information might be of interest for every user model used in a setting where the user's interest needs to be captured or the user's process of information perception and organization shall be supported [8].

But how can such a preference be elicited? We propose to use existing text, written by the target user and published in the internet, as a source of information about the user's perceptual preference. Psycholinguistic methods have been used to obtain information on the user's personality [12], moods [3], affect [15;16] and sentiments

\footnotetext{
1 In Liliana Ardissono, Tsvi Kuflik (Eds.): Advances in User Modeling - UMAP 2011 Workshops, Girona, Spain, July 11-15, 2011, Revised Selected Papers. Lecture Notes in Computer Science 7138. Springer 2012. pp. 397-401.
} 
$[1 ; 7 ; 11]$, but not yet to understand information on the user's cognitive style concerning sensory preference. We follow the tradition of lexicon based approaches such as used in opinion and sentiment mining when we look for expressions with a direct link to a sensory system, e.g., "green" or "see" as visual, and "loud" as auditory words.

\section{Method}

Our work proposes an extension to existing user models. We present a modeling technique that implicitly acquires sensory vocabulary data by analyzing forum text.

Sensory lexicon We based our lexicon of sensory vocabulary on the list of stems of sensory vocabulary collected by [9]. The list of sensory stems may be divided into 4 disjoint sets, namely the lexicon of visual vocabulary $\mathrm{L}_{\mathrm{V}}$, of auditory vocabulary $\mathrm{L}_{\mathrm{A}}$, of kinesthetic vocabulary $\mathrm{L}_{\mathrm{K}}$, and of olfactory and gustatory vocabulary $\mathrm{L}_{\{\mathrm{OG}\}}$.

Measures The use of sensory vocabulary in a document $p$ is expressed as a fourdimensional vector vprofile $(p)=[p . V, p . A, p . K, p . O G]$ based on the frequency of sensory vocabulary per sensory system. Thus, the visual component of the vector (the other components are calculated analogously) is defined as

$$
p . V=\frac{\left|\left\{t \epsilon p^{\prime} \mid v(t)=1\right\}\right|}{\mid\left\{t \epsilon p^{\prime} \mid v(t)=1 \text { or } a(t)=1 \text { or } k(t)=1 \text { or og }(t)=1\right\} \mid}
$$

where $t$ are the terms in $p$, which is $p$ modeled as a bag of words, and $v(t)$ (etc.) are the sensory indicators of the term:

$$
\text { indicator level } \begin{aligned}
s(t)= & 1 \text { if stem }(\mathrm{t}) \text { is in the lexicon of sensory vocabulary } \\
& =0 \text { in all other cases. }
\end{aligned}
$$

VAKOG profiles vprofile(p) were not only calculated for each document (forum post), but also for each author by concatenating all posts of this author to one new pseudo-document and calculating its profile as described in Equation (1). The similarity $\operatorname{vim}(p 1, p 2)$ between two posts was measured as the cosine similarity of their profiles.

Hypothesis Every user has a preference profile for sensory modalities, expressed as a profile of usage of sensory vocabulary. Hence, the $\operatorname{similarity} v \operatorname{sim}(p 1, p 2)$ of posts written by one author should be higher than to posts written by somebody else.

$\operatorname{avg}_{p 1, p 2 \epsilon \text { P, p1.author=p2.author }} v \operatorname{sim}(p 1, p 2)>a v g_{p 1, p 2 \in P, p 1 . \text { author } \neq p 2 \text {.author }} v \operatorname{sim}(p 1, p 2)$

where the $p i$ are posts, $P$ is the set of all posts, pi.author is the post's author, and vsim is the similarity between the VAKOG profiles of its two arguments. We restrict the comparison to those posts that have at least 3 VAKOG words. We decided to control for content similarity by treating the full-text similarity of two posts as a covariate. The hypothesis then is refined to "if two pairs of posts each have the same full-text similarity, the pair of the same author will have higher VAKOG similarity than the unrelated pair". Full-text similarity was operationalized as the cosine similarity between the two posts modeled as bags of words (BOW) by the WEKA ${ }^{2}$ StringToWordVector filter, weighted by TF.IDF [2].

\footnotetext{
${ }^{2}$ http://www.cs.waikato.ac.nz/ml/weka
} 
Data To test the hypothesis, we chose Richling's forum corpus [13]. It is a corpus built on posts from discussion forums on the car type BMW E30, published in the years 2000 until 2007. Its very narrow topic helps to minimize result variation due to discussion of different topics. The corpus is monolingual and consists of more than one million posts in German, each post text accompanied by information on the author, the header, the reference post, and the date. 


\section{Results: The use of sensory vocabulary in forums}

The E30 forum corpus consists of $1,053,841$ posts, written by 30,021 different authors. A detailed distribution can be found in Table 1.

Concerning our hypothesis, we applied two methods of testing.

(1) Comparison of distributions with MannWhitney's U test: Significance testing against the null hypothesis of equal distribution was calculated separately for full-text and for VAKOG similarity, comparing the hypothesis set with the set of all post pairs.

(2) To combine the full-text and VAKOG similarities, we used loglinear modeling for 3way contingency tables. The values of the three dimensions were: (i) pairwise full-text similarity, (ii) pairwise VAKOG similarity, and (iii) the 2 categorical values of the variable of interest (author- vs. non-author-post-relation). Similarity values were binned into five equalsized intervals. Results were calculated comparing all 3 tables at once and comparing pairs of 2 tables collapsed across the levels of the third.

All results for both methods of testing were statistically significant with a p-value $<.0001$. The hypotheses testing on the E30 forum corpus shows a significantly higher VAKOG similarity within the hypotheses subsets than within the set of all post pairs. These results obtained for tests (1) and (2) were consistent. Test (1) showed that VAKOG profiles of same-author posts were more similar than VAKOG profiles of different-author posts; test (2) showed that this also held if one controlled for full-text similarity. This confirms our hypothesis and leads to the following conclusion: Authors of forum posts have a tendency to use sensory expressions in similar distributions over time. Hence, that distribution can be considered as an interesting extension to user descriptions for user modeling.

\section{Conclusions and outlook}

In our research, we have proposed a new dimension for user modeling based on the use of sensory expressions. Based on findings from cognitive information processing and learning styles, we investigated the potential of the idea to analyze the use of sensory expression as an individual preference that might indicate sensory preference.

We opted for an implicit approach to data acquisition concerning the use of sensory vocabulary by means of analyzing forum text. We found that authors tend to use sensory expressions in similar distributions when writing new posts.

The obtained results are quite encouraging: Our next steps are to enlarge the corpus of sensory expression, investigate the relation between preferred sensory system(s) 
and the use of sensory expression by combining forum text analysis with user tests on sensory preference, and examine the influence of topic on the use of sensory expression. More detailed results are published in [10].

Acknowledgements Thanks a lot to Julia Richling for giving us full access to her forum corpus. Funding for this research was partly provided by the fFORTE WIT - Women in Technology Program of the Vienna University of Technology.

\section{$5 \quad$ References}

1. Andreevskaia, A., Bergler, S., Urseanu, M.: All Blogs Are Not Made Equal: Exploring Genre Differences in Sentiment Tagging of Blogs. In: Proceedings of the Int. Conf. on Weblogs and Social Media (ICWSM07), Boulder, Colorado, pp. 195-198 (2007)

2. Baeza-Yates, R., Ribeiro-Neto, B.: Modern Information Retrieval. Addison-Wesley, Harlow (1999)

3. Balog, K. de Rijke, M.: How to Overcome Tiredness: Estimating Topic-Mood Associations. In: Proceedings of the Int. Conf. on Weblogs and Social Media (ICWSM07), pp. 199-202 (2007)

4. Coffield, F., Moseley, D., Hall, E., Ecclestone, K.: Learning styles and pedagogy in post-16 learning. A systematic and critical review. LSRC, London (2004)

5. Dunn, R.: Commentary: Teaching Students Through Their Perceptual Strengths or Preferences. In: Journal of Reading 31/4, pp. 304-309 (1988)

6. Felder, R., Silverman, L: Learning and Teaching Styles In Engineering Education. In: Engr. Education, 78/7, pp. 674-681 (1988)

7. Godbole, N., Srinivasaiah, M., Skiena, S.: Large-Scale Sentiment Analysis for News and Blogs. In: Proceedings of the ICWSM07, Boulder, Colorado, pp. 219-222 (2007)

8. Heinath, M., Dzaack, J., Wiesner, A., Urbas, L.: Applications for Cognitive User Modeling. In: Conati, C., McCoy, K., Paliouras, G. (Eds.): UM 2007. LNCS, vol. 4511, pp. 127-136. Springer, Heidelberg (2007)

9. Kellner, G.: Wege der Kommunikationsoptimierung. Anwendung von NLP im Bereich der Künstlichen Intelligenz. VDM, Saarbrücken (2010)

10.Kellner, G., Berendt, B.: Extracting Knowledge about Cognitive Style. The Use of Sensory Vocabulary in Forums: A Text Mining Approach. In: Proceedings of the NLPKE 2011. IEEE press (2011, in press)

11.Mei, Q., Ling, X., Wondra, M., Su, H., Zhai, C.X.: Topic sentiment mixture: modeling facets and opinions in weblogs. In Proceedings of the international conference on World Wide Web, pp. 171-180 (2007)

12.Nowson, S.: The Language of Weblogs: A study of genre and individual differences. Doctoral Thesis. University of Edinburgh (2006)

13.Richling, J.: Die Sprache in Foren und Newsgroups. VDM, Saarbrücken (2008)

14.Sadler-Smith, E., Riding, R.: Cognitive style and instructional preferences. In: Instructional Science 27, pp. 355-371 (1999)

15.Strappavara, C., Valitutti, A., Stock, O.: The AffectiveWeight of Lexicon. In: Proceedings of the Fifth International Conference on Language Resources and Evaluation (LREC), Genoa, pp. 423-426 (2006)

16.Valitutti, A., Strappavara, C., Stock, O.: Developing Affective Lexical Resources. In: PsychNology 2/1, pp. 61-83 (2004) 